\title{
LES INDICATIONS GEOGRAPHIQUES ET LA PROTECTION DU PATRIMOINE CULTUREL IMMATERIEL. UN LIEN INEXCUSABLE
}

\author{
INDICAÇÕES GEOGRÁFICAS E PROTEÇÃO DO PATRIMÔNIO CULTURAL INTANGÍVEL. \\ UMA LIGAÇÃO INEVITÁVEL
}

GEOGRAPHICAL INDICATIONSAND PROTECTION OFINTANGIBLE CULTURAL HERITAGE. AN INEXCUSABLE LINK

\section{INDICACIONES GEOGRÁFICAS Y PROTECCIÓN DEL PATRIMONIO CULTURAL INMATERIAL. UN NEXO INEXCUSABLE}

\section{Marta Carolina Giménez Pereira ${ }^{1}$}

Licença CC BY:

Artigo distribuído sob os termos Creative Commons, permite uso e distribuição irrestrita em qualquer meio desde que o autor credite a fonte original.

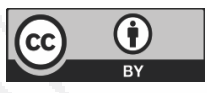

\begin{abstract}
Resumo: Os sinais característicos protegidos pela propriedade intelectual são uma possibilidade concreta de reconhecimento e desenvolvimento econômico de uma região. O título de Patrimônio Cultural Intangível da UNESCO é o meio para alcançar a representação e a preservação cultural e histórica de um país. Os dois se combinam e se encontram para atingir seus objetivos, localmente e no cenário supranacional. O texto analisará seus sucessos e fracassos de uma perspectiva de direito comparado.
\end{abstract}

Palavras-chave: Patrimônio cultural intangível; Preservação Cultural; Desenvolvimento econômico.

$1 \quad$ Docteur en droit de l'Instituto de Investigaciones Jurídicas, UNAM, Mexique. Postdoctorat en Droit de la Faculdade Meridional IMED, Brésil. Professeur chercheur auprès du PPGDireito Universidade Federal da Bahia (UFBA), Brésil. Lignes de recherche : Mécanismes efficaces de démocratie et de développement durable. Groupe de recherche : Loi, nouvelles technologies et développement. Leader du Groupe de recherche en Propriété Intellectuelle auprès de l'Universidade Federal da Bahia (UFBA), Brésil. E-mail: magipe@hotmail.com. 
Résumé: Les signes distinctifs protégés par la propriété intellectuelle sont une possibilité concrète de reconnaissance et de développement économique d'une région. Le titre de patrimoine culturel immatériel l'UNESCO est le moyen de parvenir à la représentation et à la préservation culturelle et historique d'un pays. Les deux se conjuguent et se rencontrent pour atteindre leurs objectifs, localement et sur la scène supranationale. Le texte analysera ses succès et ses échecs, dans une perspective de droit comparé.

Mots-clés: Patrimoine culturel immatériel; Préservation Culturelle; Développement économique.

Abstract: The distinctive signs protected by intellectual property are a concrete possibility of recognition and economic development of a region. The title of Intangible Cultural Heritage UNESCO is the means to achieve the representation and the cultural and historical preservation of a country. The two combine and meet each other to achieve their goals, both locally and on the supranational scenario. The text analyzes its successes and failures, from a perspective of comparative law.

Keywords: Intangible Cultural Heritage; Cultural Preservation; Economic development.

Resumen: Las señales características protegidas por la propiedad intelectual son una posibilidad concreta de reconocimiento y desarrollo económico de una región. El título de Patrimonio Cultural Inmaterial de la UNESCO es el medio para alcanzar la representación y la preservación cultural e histórica de un país. Los dos se combinan y se encuentran para alcanzar sus objetivos, localmente y en el escenario supranacional. El texto analizará sus triunfos y fracasos desde una perspectiva de derecho comparado.

Palabras-clave: Patrimonio cultural inmaterial; Preservación Cultural; Desarrollo económico.

\section{INTRODUCTION}

L'indication géographique (ci-après dénommée IG) est connue comme un actif incorporel exclusif et collectif ${ }^{2}$ qui se situe dans le droit de la propriété industrielle et couvre les sous-figures de l'indication de provenance (ci-après IP) et l'appellation d'origine (ci-après $\mathrm{AO}$ ), lesquelles constituent par conséquent des instituts de droit industriel.

Au Brésil, l'organisme administratif chargé d'accorder l'enregistrement de I'IG, à savoir I'Institut National de la Propriété Industrielle (INPI), conceptualise I'institution et déclare qu'elle permet de délimiter une zone géographique avec restriction de l'usage du nom aux producteurs et fournisseurs des services dans la région qui sont généralement organisés en entités représentatives ${ }^{3}$, telles que les

2 Welge Gonçalves, Marcos Fabrício. "Propriedade Industrial e a proteção dos nomes geográficos. Indicações geográficas, indicações de procedência e denominações de origem”. Curitiba: Juruá Editora, 2007, p. 39.

3 INPI, édition en internet : <http://www.inpi.gov.br/noticias/inpi-concede-indicacao-geografica-para-queijo-eamendoas-de-cacau>, consultée le 7 juin 2018. 
coopératives. Elle est utilisée pour identifier l'origine des produits ou des services lorsque le lieu est connu ou lorsqu'une certaine caractéristique ou qualité du produit ou du service est due à son origine.

En dépit d'une évolution législative internationale moderne et acceptable, les $\mathrm{AO}$ manquent d'un traitement systématique de la part de la doctrine qui traite d'une étude globale des antécédents, de ses statuts juridiques et des perspectives dans le futur de la figure notamment dans les Accords de libre-échange, bien que ce soit un outil fondamental et de transcendance économique et sociale dans le monde actuel où l'accréditation des qualités distinctives des produits et services sur le marché acquiert un rôle fondamental dans la concurrence et dans lequel I'AO accordée révèle une sorte d'image, une carte de présentation dans le scénario international de l'économie atteint par le pays en question et de forte poussée dans l'objectif d'augmenter les exportations de ses produits et services identifiés avec ce titre, tout en renforçant le commerce international.

D'un point de vue plus général sur le territoire européen, la régulation de ce continent met en évidence un traitement différencié des exploitations viticoles, les séparant des autres productions agro-alimentaires, bénéficiant à ces dernières d'un instrument juridique de portée européenne. En Amérique latine, un timide réveil semble s'inquiéter d'une culture de plus grande valorisation des IG qui marche au rythme des négociations des Accords de libre-échange, si à la mode aujourd'hui comme un mécanisme de consolidation des pays sur le marché international.

En ce qui concerne les IG, aussi un intérêt public se démarque, comme prévu dans le cas du Brésil dans sa Constitution nationale dans son article 5, XXIX en conformité avec les dispositions de la Loi sur la propriété industrielle du même pays, dans son article 2 .

Bien que la désignation constitutionnelle des 'auteurs d'inventions industrielles' comme les seuls sujets pour l'utilisation de ce privilège n'est pas très heureuse, la condition imposée que l'utilisation et la protection doivent coïncider avec les besoins sociaux et de développement particulières du pays mérite d'être soulignée, lesquels ne sont pas minimes parce que c'est un pays en voie de développement, sur lequel nous allons approfondir. 
Malgré les efforts juridiques déployés pour protéger la question, la législation est encore précaire et est rejetée par l'octroi du registre par l'organe administratif, I'INPI, et il y a de fortes critiques dans la doctrine. En plus d'avoir une législation malheureuse sur le sujet, d'autres problèmes se détachent, tels que l'absence de mécanismes appropriés pour la mise en œuvre des IG sur leur territoire et leur projection ou expansion à l'étranger et dans le commerce international. Il n'existe pas non plus d'organe institutionnel compétent chargé de sauvegarder et de protéger les intérêts des titulaires.

Dans un effort institutionnel considérable dans ce même pays, il a été signé le Règlement d'utilisation du nom géographique qui consiste en un document d'utilisation obligatoire pour demander le registre d'une IG et contient les règles approuvées par la communauté des producteurs ou des fournisseurs de services qui utilisera I'IG prévue. Le document décrit la typicité du produit, en soulignant les principales caractéristiques et en sauvegardant le lien avec l'origine géographique. Il existe actuellement 66 règlements sur l'utilisation des IG qui ont été enregistrés auprès de I'INPI, tant nationaux qu'étrangers. Ces règlements constituent le « Plan d'action » de l'institution susmentionnée pour l'année 2018; un plan qui vise l'efficacité opérationnelle de l'Institut.

\section{LE PATRIMOINE CULTUREL IMMATÉRIEL ET SA RELATION AVEC LES SIGNES DISTINCTIFS. UN LIEN POSSIBLE?}

A propos du thème du patrimoine culturel immatériel alimentaire et son lien avec la figure de l'IG à l'étude, il sera possible précisément en fonction de l'intérêt public qui traite intrinsèquement cette dernière figure, englobant à la fois des facteurs sociaux et technologiques, lesquels seront décrits plus tard. La particularité brésilienne de les considérer comme des facteurs conditionnant l'existence de l'octroi de la protection prévue pour les institutions énumérées dans la Loi sur la propriété industrielle, article 2, est soulignée ${ }^{4}$. Ainsi, cette fonction prévaudra toujours sur l'intérêt « égoïste et exclusif inhérent aux droits de propriété intellectuelle » 5 .

\footnotetext{
$4 \quad$ Loi n 9.279 du 14 mai 1996 (loi sur la propriété industrielle) : « Article 2. Dans l'intérêt de la société et compte tenu du développement technique et économique du pays, la protection des droits de propriété industrielle est assurée par I. la délivrance de brevets d'invention et de brevets de modèle d'utilité ; II. I'enregistrement de dessins ou modèles industriels ; III. l'enregistrement de marques; IV. la répression des fausses indications géographiques; et $\checkmark$. la répression de la concurrence déloyale ».

5 Barbosa Borges, Denis et al. « Direito da Inovação », Rio de Janeiro: Lumen luris. Deuxième Edition, 2011, p. 11.
} 
Voyons. Tout d'abord, dans le cas des IG, le fondement de leur protection et de leur contrôle du point de vue social réside dans la sécurité que les produits et services avec un IG apportent au consommateur, dans le cadre de ses droits, ainsi que la protection du produit et du producteur contre la concurrence déloyale.

Deuxièmement, on souligne le rôle qu'elle joue en tant que facteur du développement social, technologique et économique des petits producteurs, en respectant pleinement le but de la propriété industrielle de tout pays qui la contemple et la protège.

Sur le plan social international, les doctrinaires ont reconnu l'AO et en lui-même I'IG en tant qu'instruments au service de l'achèvement du Marché commun et d'une politique agricole commune, reconnaissant les deux institutions comme partisans d'une mondialisation et d'une intégration régionale, avec une perspective transnationale ${ }^{6}$.

Enfin, il est évident une conséquence de cet intérêt social reflété dans la préservation du patrimoine culturel d'un pays ou d'une région, intérêt partagé avec le titre conféré par l'Organisation des Nations Unies pour l'Éducation, la Science et la Culture (UNESCO) en sa Liste représentative qu'elle accorde au patrimoine culturel immatériel et qui vise à éviter une dépersonnalisation de celle-ci dans chaque pays demandeur.

\section{DEUX CAS: L'EXPÉRIENCE MEXICAINE ET LE BUT SOCIAL DU BRÉSIL}

Exemplifiant ce qui concerne les repas, seules les cuisines traditionnelles japonaise, française ${ }^{7}$ et mexicaine, ainsi que le régime méditerranéen (Chypre, Croatie $^{8}$, Espagne, Grèce, Italie, Maroc et Portugal), ont été déclarés patrimoine immatériel alimentaire de l'humanité par I'UNESCO ${ }^{9}$, révélant en conséquence un ensemble et une série de connaissances, rituels, traditions, pratiques, procédures et symboles d'élaboration traditionnelle spécifique, sans entrer dans le détail de

6 Ribeiro de Almeida, Alberto. "A Autonomía Jurídica da Denominação de Origem". Coimbra : Coimbra Editora, 2010, p. 610 y ss.

7 Selon I'UNESCO, la culinaire française s'agit d'un repas festif dont les convives pratiquent, pour cette occasion, l'art du " bien manger » et du " bien boire ». UNESCO, édition en internet : <https://ich.unesco.org/es/RL/lacomida-gastronomica-de-los-franceses-00437>, consultée le 6 juin 2018.

$8 \quad$ Tel est le cas du pain aux épices fabriqué dans le nord de la Croatie.

9 Parmi les requérants : la technique de conservation et d'assaisonnement coréen appelée "kimchi", le café turc aussi bien qu'une vieille méthode de vinification géorgienne appelée "kvevris". 
sa valeur nutritionnelle mais mettant en évidence des facteurs tels que la pêche et l'élevage, sa conservation, sa transformation et une méthode spécifique de cuisson, de présentation et de consommation des aliments dans un cadre d'échange social et de communication parmi les sujets concernés. Même la pratique est évaluée dans l'intention de promouvoir la consommation d'ingrédients naturels locaux qui sont transmis dans la famille de génération en génération.

L'analyse des sujets abordés me fait faire brièvement un accent sur la cuisine mexicaine, qui a un point fort dans l'utilisation d'ingrédients anciens et ancestraux, avec une utilisation abondante d'épices, et a été déclarée, comme on a noté, patrimoine culturel immatériel par I'UNESCO, désignant en 2010 la cuisine traditionnelle mexicaine comme culture communautaire ancestrale et vivante. La «Liste représentative» du patrimoine culturel immatériel l'inclut sous le nom de «Cuisine traditionnelle mexicaine : culture communautaire, ancestrale, populaire et actuelle. Le paradigme de Michoacán «. Elle a été déposée et déclarée en conséquence en tant que modèle culturel global comprenant l'agriculture, les pratiques rituelles, les anciennes compétences, les techniques culinaires et des coutumes et des modes de comportement communautaire ancestraux grâce à la participation du peuple à l'ensemble de la chaîne alimentaire traditionnelle à partir de la plantation et la récolte et jusqu'à la préparation culinaire et la dégustation. Les éléments de base du système sont: le maïs, les haricots et le chile. Les méthodes sont uniques en son genre, comme les champs de maïs connu comme «milpa» (rotation des cultures de maïs et d'autres plantes, avec brûlis de la terre) et la «chinampa» (culture d'îlots artificiels dans les zones lacustres). Les procédures pour la préparation des aliments sont très particulières, tels que la «nixtamalización» qui est composé du maïs décortiqué avec l'eau de chaux pour augmenter sa valeur nutritive. Enfin, d'autres ingrédients indigènes comme les tomates de différentes variétés, les citrouilles, les avocats, le cacao et la vanille sont ajoutés aux produits alimentaires de base. C'est ainsi que la cuisine mexicaine devienne très élaborée et chargé de symboles : la «tortilla» et les «tamales» consommés tous les jours font également partie des offres qui sont faites dans les fêtes traditionnelles comme le «Dia de Muertos». Géographiquement, dans I'État de Michoacan, comme dans d'autres localités mexicaines, il y a des groupes 
de cuisiniers et d'autres personnes qui pratiquent les traditions culinaires consacrés à l'amélioration des matières premières et de la cuisine traditionnelle. La caractéristique est que les connaissances, ainsi que les techniques, sont l'expression de l'identité communautaire et permettent de renforcer les liens sociaux et de consolider le sentiment d'identité aux niveaux national, régional et local. Ainsi, parmi les efforts qui ont été déployés au Michoacán pour préserver la cuisine traditionnelle, son importance en tant que moyen de développement durable ressort également ${ }^{10}$.

Les différents éléments qui composent cette culinaire et les différentes étapes qui l'intègrent, en particulier celle pratiquée au Michoacán, démontrent la particularité ainsi que la singularité et la spécificité de la cuisine mexicaine qui se combine dans ses éléments, en s'entrelaçant dans ses différentes étapes, dès la récolte aux techniques de préparation culinaire faite par des femmes, en passant par l'utilisation d'ingrédients ancestraux tout aussi traditionnels pour réaliser la présentation symbolique chargée de rites, tout cela même avec un tel soin de l'environnement et dans un ensemble promu par le Conservatoire de Culture Gastronomique Mexicaine lequel a mérité, comme il a été cité, la reconnaissance du patrimoine culturel immatériel accordée par I'UNESCO en 2010, selon le document d'enregistrement intitulé 5.COM 6.30. par lequel s'est prononcé à cet égard son Comité Intergouvernemental de Sauvegarde du Patrimoine Culturel Immatériel.

Bref, l'originalité de la cuisine mexicaine, sa diversité, sa capacité de cohésion sociale et de transmission des habitudes, des valeurs et des connaissances au fil du temps, donnent une sédimentation profonde dans la société et constituent l'essence de sa tradition et cela peut être largement documenté ainsi que justifié comme digne d'une reconnaissance en tant que patrimoine intangible nécessaire de protection et de préservation dans le monde de la globalisation ${ }^{11}$.

Hors le cas alimentaire, la liste représentative du patrimoine culturel immatériel de l'UNESCO, dans le cas mexicain, est intégrée par ces titres :

- Les fêtes indigènes dédiées aux morts (2008)

10 UNESCO, édition en internet : <https://ich.unesco.org/es/RL/la-cocina-tradicional-mexicana-cultura-comunitariaancestral-y-viva-el-paradigma-de-michoacan-00400>, consultée le 5 juin 2018.

11 UNESCO, ibídem, pp. 10-11. 
- Les lieux de mémoire et traditions vivantes du peuple Otomí-Chichimecas de Tolimán : la Peña de Bernal, gardienne d'un territoire sacré (2009)

- La cérémonie rituelle des Voladores (2009) ${ }^{12}$

- Les Parachicos dans la fête traditionnelle de janvier à Chiapa de Corzo (2010)

- La Pirekua, chant traditionnel des P'urhépecha (2010)

- La cuisine traditionnelle mexicaine - culture communautaire, vivante et ancestrale, le paradigme de Michoacán (2010) ${ }^{13}$

Reprenant maintenant l'intérêt public de I'IG et sa relation avec les droits des consommateurs, au sein de cet intérêt a été mentionné ci-dessus le but social qui lie ces IG avec de tels droits au Brésil ${ }^{14}$. Il trouve une base juridique constitutionnelle dans les articles 5 XXII et $170 \mathrm{~V}$ ainsi que dans le Code de protection du consommateur dans ses articles 2, 6 et 81 qui protègent les droits diffus, collectifs et individuels homogènes des consommateurs. La mise en place d'un mécanisme juridique de protection et de contrôle des IG permet de protéger ces droits ${ }^{15}$.

Les problèmes qui sont constamment causés par les aliments font que la revendication par les consommateurs soit fréquente en ce qui concerne la qualité, la disponibilité et l'étiquetage des informations sur le produit et son origine, y compris le processus d'élaboration, étant tous les facteurs déterminants lors du choix et de l'acquisition, ce qui a amené les producteurs à sensibiliser davantage à ces demandes du public acheteur, surtout les petits producteurs, qui ont su adapter leur production aux nouveaux besoins du marché consommateur, devenant de plus en plus demandés et leurs produits plus valorisés économiquement dans la compréhension que leurs consommateurs pouvaient plus facilement connaître l'origine du produit, la méthode de fabrication et quels sont les types de matières premières utilisées dans la composition, avec la conséquence que les clients sont devenus plus confiants par des produits ayant ces caractéristiques ${ }^{16}$ générant une

12 Les "voladores de Papantla" ont subi un cas récent de violation de leurs droits parce que l'image a été utilisée par la célèbre Cervecería Moctezuma pour leurs marques, sans le consentement d'eux.

13 Le mariachi et la charrería sont aussi des points d'intérêt et d'étude.

14 Dans le présent article, il existe un désaccord dans l'identification de l'intérêt social avec l'intérêt public parce que, à mon avis, le premier est inséré dans le second, mais pas l'inverse.

15 Carvalho da Rocha Porto, Patrícia. "Quando a Propriedade Industrial...", op. cit., p. 178.

16 Ibídem, p. 179. 
chaîne de production continue et stable et un modus vivendi traduit en une sorte de coexistence directe entre le producteur et le consommateur, caractérisée par la confiance et la proximité entre les deux parties, un traitement différencié, la crédibilité et la fiabilité et, par conséquent, l'obtention d'un résultat supérieur à la production, aux soins et à la commercialisation de masse, y compris la livraison en mains propres au consommateur, dans certains cas, par le producteur luimême. Ce fut le cas de pays pionniers tels que la France, l'Italie, l'Angleterre, les États-Unis et, en émergence, au Brésil et au Mexique, comme décrit.

En second lieu, a été introduite l'idée de fonction exercée par l'IG en tant que facteur de développement social, technologique et économique des petits producteurs et des petites exploitations agricoles en gardant à l'esprit qu'ils ont eu des revers comme les mises à jour qui ont souffert progressivement et en continu l'agro-industrie avec le progrès technologique dans ce domaine, ainsi que le progrès de la science, de plus en plus professionnelle, industrialisée, en vue de devenir plus globale. Les petits agriculteurs ont été contraints de prendre des petites mesures, telles que: la concentration des ressources sur les marchés de niche; la spécialisation de ce qu'ils produisent; la connexion de la production avec la région d'origine et avec des facteurs naturels et humains; plus d'informations sur la méthode de fabrication; les informations des composants et l'addition des valeurs culturels dans la fabrication de produits afin d'avoir un résultat unique et d'entrainer une histoire, avec une valeur économique ajoutée éveillant l'intérêt des consommateurs en raison d'être uniques, différents et spéciaux. Comme résultat de ces caractéristiques de distinction, protection et contrôle gagnées par le titre d'IG, on a obtenu que les plus petites propriétés productrices, avant dévalorisés, deviennent des zones de grande croissance économique, sociale et technologique ${ }^{17}$, selon les dispositions légales énoncées dans le pays, ainsi que la tendance mondiale avec une chaîne en évolution, auto-motivée constamment et qui se traduit, enfin, par l'amélioration de la qualité de vie en raison d'être une sorte d'influence sur l'amélioration de la santé de la région ainsi que du tourisme et des conditions de logement de ses habitants.

La dernière des fonctions sociales de I'IG a un lien avec la préservation du patrimoine culturel d'un pays ou d'une région, où il trouve convergence et 17 Ibídem, p. 181. 
homonymie des intérêts avec le titre de patrimoine culturel immatériel qui a été mentionné auparavant. Le patrimoine culturel national est répertorié comme une garantie prévue aux articles 215 et 216 de la Constitution fédérale brésilienne qui signalent la protection et la valorisation par des lois spécifiques, ainsi que l'accès par sa population, et il est de la responsabilité du gouvernement d'assurer cette protection et son accès.

La même norme établit que le patrimoine culturel est cette manière de créer, de faire et de vivre dans un lieu spécifique et qu'il appartient à l'État et aux citoyens de le promouvoir et de le préserver. Les IG sont un moyen. La déclaration du patrimoine culturel immatériel par I'UNESCO est ainsi un autre moyen de préserver à la fois le patrimoine et son histoire pour qu'ils restent vivants.

Selon les mots de Carvalho da Rocha Porto, les IG, résumant et de manière spécifique, remplissent une fonction sociale lorsqu'elles servent le but pour lequel elles ont été conçues, à savoir : "...Préserver le patrimoine culturel et historique, la culture, l'identité et la manière de créer, de faire et de vivre des peuples de la région protégés par le signe distinctif $»^{18}$.

Quant au patrimoine culturel immatériel, il se produit de la même manière et l'intention de sa préservation circule dans le même sens : s'incarner dans une mémoire vivante, dans une action constante de soin, de pratique et de préservation. Cependant, les potentialités de ces signes distinctifs en tant que véhicule pour protéger ce patrimoine, ses véritables efficacités, doivent être analysées.

Pourtant, le retard excessif dans le traitement de la procédure d'un tel titre devantI'UNESCO aété dénoncé, institution quiactuellementadmetseulement deux demandes par $a^{19}{ }^{19}$. D'autre part, le titre conféré ne sert qu'à une reconnaissance, sans impliquer ou stimuler certain bénéfice économique pour le propriétaire et sans assurer les garanties qu'il semble conférer, mais au contraire, il se limite à des exigences strictes pour sa conservation. En fait, I'Organisation Mondiale de la Propriété Intellectuelle (OMPI) est la seule institution qui s'est préoccupée du profit et de la promesse de prospérité économique vis-à-vis des détenteurs de 18 Ibídem, pp. 186-187.

19 Le Panama a choisi d'approcher l'OMPI et, par conséquent, l'institution a envoyé des experts spécialisés dans ce domaine pour assurer la médiation et la protection devant I'UNESCO. Voir en UNESCO, "Panamá, Ciudad Creativa Gastronómica de la UNESCO", édition en internet : <http://www.unesco.org/new/es/media-services/single-view/ news/panama_unesco_creative_culinary_city/>, consultée le 28 juin 2018. 
signes distinctifs et du patrimoine culturel immatériel lequel, selon les fonctions de I'UNESCO, il se tournerait plutôt et simplement vers la préservation, la nonextinction de l'objet protégé. Il existe en fait un Comité intergouvernemental à I'OMPI chargé de cette tâche.

Un autre problème est l'existence majoritaire de la doctrine sociologique et anthropologique mais non juridique, spécialisée dans la propriété intellectuelle sur le patrimoine culturel immatériel. Peut-être que le moyen serait de ne pas rester dans le schéma et chercher une protection alternative qui va au-delà, avec des directives réglementaires convergentes entre les deux figures et contemplatives ainsi des points de vue plus encourageants pour la commercialisation des produits et services protégés par l'un ou l'autre titre.

Le cas du «Code Organique de l'Économie Sociale des Connaissances», mieux connu sous le nom de "Código Ingenios» de l'Equateur, sert d'exemple de paradigme. Un code pionnier démocratiquement construit par différents acteurs de la société, couvre onze principes, dont le dernier est celui qui nomme les peuples et les nationalités avec le droit à leur connaissance : le savoir traditionnel est également source de richesse pour la communauté scientifique et, par conséquent, les peuples et les nationalités auront le droit de décider leurs connaissances $^{20}$.

\section{UNE DÉGUSTATION BRÉSILIENNE : LA « CACHAÇA » ET L' « ACARAJÉ »}

Des nombreuses critiques dans la doctrine du décret présidentiel qui a institué la «Cachaça» comme IG, pour l'ambiguïté qui présente, reprochent aussi d'être une législation précaire depuis longtemps en raison de l'absence de réglementation en plus d'un manque de mécanismes efficaces de mise en œuvre de l'IG sur le territoire et sa nulle projection ou expansion à l'étranger et dans le commerce international.

Ainsi, en vertu du Décret 4602, en 2001, les expressions «Cachaça», «Brasil» et «Cachaça do Brasil» ont été définies comme IG afin de lutter contre l'utilisation abusive de telles expressions sur le marché international, conformément aux

20 SENESCYT, édition en internet : <https://www.educacionsuperior.gob.ec/wp-content/uploads/downloads/2015/06/ ingenios.pdf>, consultée le 11 juin 2018. 
dispositions de l'Accord sur les ADPIC et la propre législation nationale. Elle a été réglementée pour son utilisation seulement en octobre 2016.

La critique réside principalement dans le non-respect des dispositions de la loi sur la propriété industrielle nationale, la Loi 9279 de 1996, puisque l'expression ne fait référence à aucune région géographique.

D'autre part, il n'a pas été accordée par l'organisme administratif habilité à cet effet, à savoir l'INPI, à la demande des producteurs ou fournisseurs de services. Ainsi, dans ce cas, il semble que la voie choisie était contraire : préalablement on protège l'expression et c'est après que les potentiels titulaires sont nommés pour l'utiliser ${ }^{21}$. Par conséquent, la protection n'a pas été strictement selon les propres termes de propriété intellectuelle, et encore moins d'une $A O$, dans une stratégie peu judicieuse qui ne semble pas attirer ni une plus grande demande ni la confiance des consommateurs au sujet de leur origine. Le nom enregistré menace également être utilisé pour tout sorte de rhum produit dans une région quelconque au Brésil, c'est à dire, il risque d'être employé d'une manière générale.

Dans la région nord-est du Brésil, ses potentiels IG parmi lesquels on dénombre l'artisanat, comme la serviette en fibre de noix de coco et dentelle d'Alagoas, et leurs aliments et boissons typiques, comme le "Cupulate», les liqueurs exotiques et la « Cocada baiana » ils ajoutent une valeur incalculable au patrimoine brésilien.

Dans le cas de I' "Acarajé », recette typique de l'état brésilien de Bahia, il intègre la «Liste Auxiliaire de Produits» de l'INPI. Il devient une fusion de la culture africaine avec le nord-est du Brésil. Sa particularité est d'être le résultat des traditions ancestrales liées aux traditions religieuses afro-brésiliennes, parmi lesquelles le candomblé, issu de causes mythologiques ancestrales liées à l'offrande aux dieux, faisant partie de l'ethnographie de la région décrite, avec des particularités en conséquence anthropologiques, sociales, culturelles, sociologiques et de syncrétisme religieux. Actuellement, il est consommé avec une autre nomenclature en Afrique de l'Ouest, avec de légères adaptations. Introduit par les arabes en Afrique, il arrive au Brésil pendant la traite atlantique des esclaves.

21 Locatelli, Liliana. "Indicações geográficas. A proteção jurídica sob a Perspectiva do Desenvolvimento Econômico". Curitiba : Juruá, 2008, p. 251. 
La façon dont il est présenté a également ses propres caractéristiques, car il est vendu dans un conteneur appelé «tabuleiro da baiana ». Certains de ses ingrédients sont typiques de la région de Bahia mais pas tous de sorte que, à ce jour, I' « Acarajé » ne bénéficie d'aucune protection de I'UNESCO, non plus en tant que signe distinctif en vertu des réglementations sur la propriété intellectuelle en vigueur au Brésil| ${ }^{22}$. Cependant, le déni de sa condition sous de simples arguments est en doute.

La Liste représentative de I'Unesco du patrimoine culturel immatériel du Brésil est composée de :

- Le Círculo de Capoeira (2014)

- Le Círio de Nazaré ou la procession de l'image de Notre-Dame de Nazareth dans la ville de Belém, Pará (2013)

- Le Frevo ou l'art du spectacle de carnaval de Recife, Pernambuco (2012)

- Le Yaokwa ou le rituel du peuple Enawene nawe pour le maintien de l'ordre social et cosmique de Mato Grosso (2011)

- Le musée vivant du Fandango (2011)

- Les expressions orales et graphiques du Wajapi de l'État d'Amapá (2008)

- La Samba de Roda du Recôncavo de Bahia (2008)

Chaque année, le Programme national du patrimoine immatériel du Brésil appelle à un concours national de projets en vue de favoriser et de soutenir les initiatives et les pratiques de la société brésilienne liées à la sauvegarde du patrimoine culturel immatériel. Les projets doivent inclure la participation de la communauté et des groupes intéressés, promouvoir l'intégration sociale et améliorer les conditions de vie des créateurs et des gardiens de ce patrimoine, ainsi que respecter les droits individuels et collectifs. La sélection des projets est effectuée par le Département du patrimoine immatériel de l'Institut national du patrimoine historique et artistique (IPHAN) basé à Brasilia et est réalisée par un comité de spécialistes du pays. En bref, il s'agit d'un modèle de financement et de promotion des initiatives de la société civile visant à sauvegarder le patrimoine

22 Pas tous ses ingrédients sont produits exclusivement dans la région et ne tiennent pas compte du facteur de l'environnement durable. En outre, la recette peut varier en fonction des agrégats (carurú, vatapá, crevettes frites et pâte de poivre malagueta frite dans l'huile dendé). 
culturel immatériel ${ }^{23}$.

Fait et conduit correctement, ce Programme semble être un autre moyen d'obtenir une plus grande protection du sujet dans un pays qui mérite de jouir de plus nombreux titres auprès de I'UNESCO, comme c'est le cas au Brésil.

\section{CONSIDÉRATIONS FINALES}

L'analyse effectuée tout au long de cet article permet de déduire que la protection accordée par les signes distinctifs étudiés avec leurs différentes régions potentiellement enregistrables a une force centrifuge qui affecte la figure du patrimoine culturel immatériel, qui n'est que la réflexion sensible des terrains qui font partie de la richesse nationale autochtone d'une nation. Nous sommes face à un double axe et une tâche : la promotion de l'économie et la protection et la mise en évidence du patrimoine culturel, historique et même environnemental d'un pays.

Le problème réside dans le fait que pendant des années a été oubliée la situation d'avantage concurrentiel accordé par l'IG comme figure typique de la propriété intellectuelle et son implication intrinsèque de catapulter les pays par une offre non seulement de produits qualifiés - avec des particularités qu'en plus agissent comme une «bannière» pour présenter la richesse qui les identifie dans le commerce international et la propriété industrielle typique -, mais également en agissant comme une voie efficace et interdépendant avec le patrimoine culturel immatériel, dans une sphère et un scénario supranationaux.

Les IG sont un moyen de parvenir à un meilleur positionnement économique et géographique et la déclaration expresse du patrimoine culturel immatériel par I'UNESCO est le moyen de préserver à la fois le patrimoine et l'histoire d'une région, afin qu'ils restent vivants. Néanmoins, les potentialités des signes distinctifs en tant que véhicule pour protéger tel patrimoine, c'est-à-dire leur véritable efficacité, doivent être analysées.

Comme décrit, une IG implique une garantie de qualité, mais aussi un puissant outil de positionnement dans le domaine du commerce extérieur. Malgré les efforts juridiques déployés pour protéger cette institution, dans le cas du 23 UNESCO, édition en internet : <https://ich.unesco.org/es/estado/brasil-BR?info=elementos-en-las-listas>, consultée le 11 juin 2018. 
Brésil, la législation n'est toujours pas très heureuse et est soutenue par l'octroi de l'enregistrement par l'organe administratif, I'INPI, avec de fortes critiques doctrinales. En outre, d'autres problèmes se détachent, comme l'absence de mécanismes de mise en œuvre réussis sur son territoire et son exploitation, sa projection et son expansion dans le commerce international, malgré les excellents résultats obtenus par les détenteurs des quelques registres nationaux existants. Il n'y a pas non plus d'organisme institutionnel compétent chargé de veiller aux intérêts des titulaires, lesquels sont liés au strict respect des conditions existantes et des caractéristiques spécifiques en vertu desquelles I'IG a été accordée pour un produit ou un service donné.

Le plus remarquable est sans doute la dernière des fonctions sociales de I'IG telle qu'elle a été décrite et qui concerne la préservation du patrimoine culturel d'un pays ou d'une région, point où elle trouve convergence et homonyme d'intérêts avec le titre de Patrimoine culturel immatériel à l'étude. Le patrimoine culturel national est répertorié comme une garantie prévue aux articles 215 et 216 de la Constitution fédérale brésilienne qui prévoit la protection et la valorisation par des lois spécifiques, ainsi que l'accès par sa population. Ainsi, la norme établit que le patrimoine culturel est cette manière de créer, de faire et de vivre dans un lieu spécifique et correspond donc à la fois à l'État et aux citoyens le développer et le préserver à travers des politiques publiques de promotion sous protection constitutionnelle.

En ce qui concerne spécifiquement le patrimoine culturel immatériel, il se produit de la même manière et l'intention de sa préservation circule dans le même sens que les signes distinctifs : s'enfermer dans une mémoire vivante, dans une action constante de soin, de pratique et de préservation.

La critique soutient la faible admissibilité et le retard excessif dans le traitement de la procédure d'un tel titre devant I'UNESCO, outre le fait que le titre conféré ne sert qu'à la reconnaissance, sans impliquer ou stimuler aucun avantage économique pour le titulaire et sans veiller aux garanties qui semble conférer, exigeant plutôt des conditions strictes pour sa conservation. Du point de vue économique, seule l'OMPI a été institutionnellement soucieuse du profit et de la promesse de prospérité pour les détenteurs de signes distinctifs et du patrimoine culturel immatériel, lequel, à partir des fonctions de I'UNESCO, se 
tournerait plutôt vers une préservation, une non-extinction.

Un autre problème est la doctrine précaire spécialisée dans la propriété intellectuelle sur le patrimoine culturel immatériel. Peut-être le chemin consiste$\mathrm{t}$-il à ne pas s'insérer dans un schéma traditionnel et à chercher une protection alternative avec des orientations normatives convergeant entre les deux figures, contemplatives de points de vue plus encourageants pour la commercialisation de produits et services protégés par l'un titre ou l'autre titre.

Enfin, le travail d'un Programme national du patrimoine immatériel, réalisé correctement, semble être un autre moyen de parvenir à une meilleure protection du patrimoine culturel immatériel, en particulier dans des pays qui méritent d'avoir plus de titres devant I'UNESCO, comme il est le cas des pays d'Amérique latine comme le Mexique et le Brésil, prenant, pourquoi pas, l'exemple des pays européens qui ont déjà gagné plus de terrain à cet égard.

L'objectif du développement d'une nation est un fondement du rang constitutionnel et, par conséquent, la performance publique dans la promotion des institutions décrites est justifiée comme outil pour rendre ce but viable.

\section{RÉFÉRENCES DES SOURCES CITÉES}

Barbosa Borges, Denis et al. « Direito da Inovação », Rio de Janeiro: Lumen Iuris. Deuxième Edition, 2011.

BRÉSIL, Loi n 9.279 du 14 mai 1996 (loi sur la propriété industrielle), édition en internet : <http://www.wipo.int/wipolex/fr/details.jsp?id=515>, consultée le 29 juin 2018.

Carvalho da Rocha Porto, Patrícia. "Quando a Propriedade Industrial representa qualidade: Marcas Coletivas, Marcas de Certificação e Denominações de Origem". Río de Janeiro : Lumen Iuris, 2001.

CONVENTION POUR LA SAUVEGARDE DU PATRIMOINE CULTUREL IMMATÉRIEL. COMITÉ INTERGOUVERNEMENTAL DE SAUVEGARDE DU PATRIMOINE CULTUREL IMMATÉRIEL. Cinquième session Nairobi, Kenya novembre 2010. DOSSIER DE CANDIDATURE $N^{\circ} 00400$ POUR L'INSCRIPTION SUR LA LISTE REPRESENTATIVE DU PATRIMOINE CULTUREL IMMATERIEL EN 2010, disponible en internet : <file:///C:/Users/Usuario/Downloads/07530-FR\%20(1).pdf>, consulté le 6 juin 2018.

INPI, édition en internet : <http://www.inpi.gov.br/menu-servicos/indicacao-geografica>, consultée le 7 juin 2018. 
INPI, édition en internet: <http://www.inpi.gov.br/noticias/inpi-concede-indicacaogeografica-para-queijo-e-amendoas-de-cacau>, consultée le 7 juin 2018.

INPI, édition en internet: <http://www.inpi.gov.br/menu-servicos/indicacao-geografica/ regulamento-de-uso-das-indicacoes-geograficas>, consultée le 4 juin 2018.

INPI, édition en internet: <file:///C:/Users/Usuario/Downloads/copy3_of_ LISTACOMASINDICAESDEPROCEDNCIACONCEDIDAS.At24Abr2018.pdf >, consultée le 7 juin 2018.

IPHAN, édition en internet: <http://portal.iphan.gov.br/pagina/detalhes/74>, consultée le 11 juin 2018.

Locatelli, Liliana. "Indicações geográficas. A proteção jurídica sob a Perspectiva do Desenvolvimento Econômico". Curitiba: Juruá, 2008.

López Benítez, Mariano. "Las denominaciones de origen". Barcelona: Cedecs, 1996.

López Benítez, Mariano. "Del estatuto del vino a las leyes del vino: un panorama actual y de futuro de la ordenación vitivinícola de España". Madrid: S.L. Civitas Ediciones, 2004.

Ribeiro de Almeida, Alberto. "A Autonomía Jurídica da Denominação de Origem". Coimbra: Coimbra Editora, 2010.

Secretaría de Cultura, INAH Mediateca, édition en internet: < http://www.mediateca.inah.gob. mx/islandora_74/islandora/object/disco\%3A41>, consultée le 5 juin 2018.

SENESCYT, édition en internet: <https://www.educacionsuperior.gob.ec/los-once-principiosde-ingenios/>, consulté le 11 juin 2018.

SENESCYT, édition en internet: < https://www.educacionsuperior.gob.ec/wp-content/uploads/ downloads/2015/06/ingenios.pdf>, consultée le 11 juin 2018.

SIC MEXICO, édition en internet : <http://sic.gob.mx/ficha.php?table=frpintangible\&table_ id=260>, consultée le 5 juin 2018 .

UNESCO. Informe ITH/10/5.COM/CONF.202/5 Rev. Annexe 1, édition en internet : <https:// ich.unesco.org/doc/src/ITH-10-5.COM-CONF.202-5\%20Rev.-FR.pdf> consultée le 5 juin 2018.

UNESCO, édition en internet: <https://ich.unesco.org/es/RL/la-comida-gastronomica-de-losfranceses-00437>, consultée le 6 juin 2018.

UNESCO, édition en internet: <https://ich.unesco.org/es/RL/la-cocina-tradicional-mexicanacultura-comunitaria-ancestral-y-viva-el-paradigma-de-michoacan-00400>, consultée le 5 juin 2018. 
UNESCO, édition en internet: <file:///C:/Users/Usuario/Downloads/04955.pdf>, consultée le 6 juin 2018.

UNESCO, édition en internet: <file:///C:/Users/Usuario/Downloads/07530-FR\%20(1).pdf>, consultée le 6 juin 2018.

UNESCO, édition en internet: <https://ich.unesco.org/es/estado/mexico-MX?info=informeperiodico\#pr-2018-2018>, consultée le 6 juin 2018.

UNESCO, édition en internet: <https://ich.unesco.org/es/estado/brasil-BR?info=elementosen-las-listas>, consultée le 11 juin 2018.

UNESCO, "Panamá, Ciudad Creativa Gastronómica de la UNESCO", édition en internet: < http:// www.unesco.org/new/es/media-services/single-view/news/panama_unesco_creative_ culinary_city/>, consultée le 28 juin 2018.

Vivez, Jacques. « Traité des appellations d'origine, législation, règlementation, jurisprudence ». Paris: R. Pichon \& R. Durand-Auzias, 1943.

Welge Gonçalves, Marcos Fabrício. "Propriedade Industrial e a proteção dos nomes geográficos. Indicações geográficas, indicações de procedência e denominações de origem". Curitiba: Juruá Editora, 2007. 
